\title{
Model of an Atom by Analogy with the Transmission Line
}

\author{
Milan Perkovac \\ The First Technical School Tesla, Zagreb, Croatia \\ Email: milan@drivesc.com \\ Received March 24, 2013; revised April 27, 2013; accepted May 24, 2013
}

Copyright (C) 2013 Milan Perkovac. This is an open access article distributed under the Creative Commons Attribution License, which permits unrestricted use, distribution, and reproduction in any medium, provided the original work is properly cited.

\begin{abstract}
Model of an atom by analogy with the transmission line is derived using Maxwell's equations and Lorentz' theory of electrons. To be realistic such a model requires that the product of the structural coefficient of Lecher's transmission lines $\sigma$ and atomic number $Z$ is constant. It was calculated that this electromechanical constant is 8.27756 , and we call it structural constant. This constant builds the fine-structure constant $1 / \alpha=137.036$, and with permeability $\mu$, permittivity $\varepsilon$ and elementary charge $e$ builds Plank's constant $h$. This suggests the electromagnetic character of Planck's constant. The relations of energy, frequency, wavelength and momentum of electromagnetic wave in an atom are also derived. Finally, an equation, similar to Schrödinger's equation, was derived, with a clear meaning of the wave function, which represents the electric or magnetic field strength of the observed electromagnetic wave.
\end{abstract}

Keywords: Lecher Transmission Line; Lorentz' Theory of Electrons; Maxwell's Equations; Model of Atom; Planck Constant; Structural Constant; Transverse Mass; Wave Equation

\section{Introduction}

A hundred years ago, classical physics, with Newton's mechanics and Maxwell's theory, couldn't explain determined properties of atoms [1]. For that reason Maxwell's equations are neglected in modern physics, despite the fact that matter is composed of electrically charged particles, and that static and current electricity are in complete harmony with Maxwell's equations. The main motive of this paper is to show that the atom can be explored using Maxwell's equations. Moreover, I want to show that, in addition to Maxwell's equations and Lorentz' theory of electrons for the basic research of the atom, nothing more is needed. By using Maxwell's theory differential equations of the electromagnetic wave in any space are derived. This space could also be the space within the atom. These differential equations have the same form as the differential equations of wave on the parallel-wire transmission line, on the so-called Lecher's line [2]. Therefore, this electromagnetic wave, with the same differential equations, is treated in analogy with the wave on Lecher's line. Here I show a model of atom [3], where structural coefficient of Lecher's transmission line, corresponding to a certain atom, multiplied by their atomic number, appears as a structural constant 8.27756, which is a universal constant. This constant builds the fine structure constant, and with other constants builds
Planck's constant [4]. The energy and momentum of the electromagnetic wave can be determined using Maxwell's and Lorentz theory. This paper makes it possible to eliminate some disadvantages of classical physics mentioned at the beginning.

The idea of this article is that it is not possible to make the same thing in two distinctly different ways in nature. Therefore an electromagnetic wave that originates in the atom and the electromagnetic wave in the macro world have common ground. Due to identical differential equations a linking of electromagnetic wave in atoms and wave on the transmission lines is possible.

This linking of waves in atoms and waves on the transmission line is carried out using the parameters of atoms and transmission lines, as well as through their energy.

Researching electromagnetic energy from transmission lines it was determined that this energy can be expressed as linearly proportional to the frequency of oscillation of its own $L C$ circuit. Proportionality factor leads to structural constants and action constants. Structural constant is introduced so as to make action constant independent of $L C$ circuit natural frequency. Here, in a unique way, we can determine the value of the action constant. It's shown that action constant is equal to Planck's constant $h$.

Then we determine the frequency of the wave in the atom, the wavelength, phase velocity and momentum of 
the wave.

Knowledge of these quantities allows determination of the properties of space in which these phenomena take place.

Finally, we can determine the wave equation according to which all of these phenomena are governed by investigating the atom.

\section{The Atom and the Transmission Line}

Using equations of Maxwell's theory, [5], it is possible to obtain the states of the electric field (vector $\boldsymbol{E}$ ) and magnetic field (vector $\boldsymbol{H}$ ) in any space, even within the atoms. The mathematical description of these states are presented with two second-order linear partial differential equations (wave equations) $[1,6]$ :

$$
\nabla^{2} \boldsymbol{E}-\frac{1}{u_{\mathrm{em}}^{2}} \frac{\partial^{2} \boldsymbol{E}}{\partial t^{2}}=0 ; \nabla^{2} \boldsymbol{H}-\frac{1}{u_{\mathrm{em}}^{2}} \frac{\partial^{2} \boldsymbol{H}}{\partial t^{2}}=0
$$

where $\nabla^{2}$ is del-squared, $\frac{\partial^{2}}{\partial x^{2}}+\frac{\partial^{2}}{\partial y^{2}}+\frac{\partial^{2}}{\partial z^{2}}, t$ is time and $u_{\mathrm{em}}$ is phase velocity of the electromagnetic wave, which dependents on the medium, i.e., $u_{\mathrm{em}}=1 / \sqrt{\varepsilon \mu}$; $\varepsilon=\varepsilon_{\mathrm{r}} \varepsilon_{0}$ is permittivity, $\varepsilon_{\mathrm{r}}$ is relative permittivity, $\varepsilon_{0}$ is permittivity of free space, $\mu=\mu_{\mathrm{r}} \mu_{0}$ is permeability, $\mu_{\mathrm{r}}$ is relative permeability and $\mu_{0}$ is permeability of free space.

The same form of differential equations as previous, but only in one dimension, [2], is also present on the parallel-wire transmission line, called Lecher's line, consisting of a pair of ideal conducting parallel wires of radius $\rho$, separated by $\delta$, wherein the ratio $\delta / \rho=\chi$ :

$$
\frac{\partial^{2} u}{\partial z^{2}}-L^{\prime} C^{\prime} \frac{\partial^{2} u}{\partial t^{2}}=0 ; \frac{\partial^{2} i}{\partial z^{2}}-L^{\prime} C^{\prime} \frac{\partial^{2} i}{\partial t^{2}}=0,
$$

where $L^{\prime}=\mu(\ln \chi+1 / 4) / \pi$ is inductance of Lecher's line per unit length and $C^{\prime}=\pi \varepsilon / \ln \left[\chi / 2+\sqrt{(\chi / 2)^{2}-1}\right]$ is its capacitance per unit length, $u$ is voltage at the entrance to the element $\mathrm{d} z$ of Lecher's line, and $i$ is electrical current at the entrance to the element $\mathrm{d} z$ of Lecher's line $[2,4]$.

The same form of differential equations for example those in the atom and on the Lecher's line, allows finding unknown solutions in the atom using known solutions on Lecher's line. In this case, the voltage $u$ on the Lecher's line is analogous to the electric field $E$, while the electric current $i$ on the Lecher's line is analogous to the magnetic field $H$. Therefore, the voltage and current on Lecher's line behaves the same way as the electromagnetic wave in the atom. The analogy between the wave on the transmission line and electromagnetic wave will be completed when we put that energy into transmission line, then it is equal to the energy of the electromagnetic wave.

On one hand, such transmission line can be regarded as a limiting case of an $L C$ network with infinitely small capacitors and inductors [7]. If all small capacitors of the network are put on the open end $(C)$, and all small inductances are put on the shot-circuited end $(L)$ of Lecher's line, then the natural frequency of such oscillatory circuit is $v=1 /(2 \pi \sqrt{L C})$, [8].

\section{Electromagnetic Energy in an Atom and on the Transmission Line}

We can determine the amount $E_{\text {em }}$ of electromagnetic energy of a wave and bring that energy to the $L C$ circuit formed in the manner described by Lecher's line, [9]: $E_{\text {em }}=1 / 2 \hat{Q}_{\mathrm{C}}^{2} / C$, where $\hat{Q}_{\mathrm{C}}$ is a maximum charge on the said capacitor $C$.

On the other hand, however, in force equation according to Newton's second law, $F=m a$, we substitute aceleration $a$ from $a=v^{2} / r, F$ from Coulomb's law, $q Q / 4 \pi \varepsilon r^{2}$, and $m$ from transverse mass of the electron according to Lorentz' theory. Because the acceleration is at right angles with respect to the velocity, this transverse mass of the electron is $m / \sqrt{1-\beta^{2}}$, [10]. Therefore we obtain:

$$
\frac{m v^{2}}{r \sqrt{1-\beta^{2}}}=\frac{|q Q|}{4 \pi \varepsilon r^{2}} ; r=\frac{|q Q| \sqrt{1-\beta^{2}}}{4 \pi \varepsilon m c^{2} \beta^{2}},
$$

where $r$ is the radius of the circular orbit of the electron in an atom, $q$ is the charge of the electron $(q=-e), Q$ is the charge of the nucleus $(Q=Z e), Z$ is atomic number, $m$ is the electron rest mass, $c$ is the speed of light in vacuum, $\beta=v / c$, where $v$ is the electron velocity.

The kinetic energy of electron is

$K=m c^{2} / \sqrt{1-\beta^{2}}-m c^{2}$, [9]. Using Equation (3) and noting that an electron is of opposite charge of the nucleus, than the potential energy of electron is

$U=q Q / 4 \pi \varepsilon r=-m c^{2} \beta^{2} / \sqrt{1-\beta^{2}}$. The total mechanical energy of the electron $E_{\mathrm{T}}=K+U=-m c^{2}\left(1-\sqrt{1-\beta^{2}}\right)$, according to the law of conservation of energy is equal to the negative emitted electromagnetic energy,

$E_{\text {em }}=m c^{2}\left(1-\sqrt{1-\beta^{2}}\right)=e V,[4]$; here $V$ is the potential difference, which passes an electron from the point of reference potential, to the potential of the point at which an electron is currently located, i.e., $E_{\mathrm{T}}=K+U=-E_{\mathrm{em}}=-e V$.

According to $E_{\mathrm{em}}=m c^{2}\left(1-\sqrt{1-\beta^{2}}\right)$ we can write: 


$$
\sqrt{1-\beta^{2}}=1-\frac{E_{\mathrm{em}}}{m c^{2}} ; \beta=\sqrt{2 \frac{E_{\mathrm{em}}}{m c^{2}}\left(1-\frac{E_{\mathrm{em}}}{2 m c^{2}}\right)} .
$$

Now, the radius $r$ in Equation (3) we write:

$$
\begin{gathered}
r=|q Q|\left(1-E_{\mathrm{em}} / m c^{2}\right) /\left[8 \pi \varepsilon E_{\mathrm{em}}\left(1-E_{\mathrm{em}} / 2 m c^{2}\right)\right], \text { that is } \\
E_{\mathrm{em}}=\frac{1}{2} \frac{|q Q|}{4 \pi \varepsilon r} \frac{1-E_{\mathrm{em}} / m c^{2}}{1-E_{\mathrm{em}} / 2 m c^{2}}=\frac{1}{2} \frac{\hat{Q}_{\mathrm{C}}^{2}}{C} .
\end{gathered}
$$

This single Equation (5) has two unknown sizes, i.e. parameter $C$ and variable $\hat{Q}_{\mathrm{C}}$. With the help of Diophantine equations we obtain one of the solutions: $C=4 \pi \varepsilon r$, and $\hat{Q}_{\mathrm{C}}^{2}=|q Q|\left(1-E_{\mathrm{em}} / m c^{2}\right) /\left(1-E_{\mathrm{em}} / 2 m c^{2}\right)$, [4].

We can transform now the Equation (5) of the electromagnetic energy in $L C$ circuit [4]:

$$
\begin{aligned}
E_{\mathrm{em}} & =\frac{1}{2} \frac{\hat{Q}_{\mathrm{C}}^{2}}{C}=\frac{1}{2} \frac{\pi}{\pi} \frac{\hat{Q}_{\mathrm{C}}^{2}}{\sqrt{C} \sqrt{C}} \frac{\sqrt{L}}{\sqrt{L}}=\pi \sqrt{\frac{L}{C}} \frac{\hat{Q}_{\mathrm{C}}^{2}}{2 \pi \sqrt{L C}} \\
& =\pi Z_{\mathrm{LC}} \hat{Q}_{\mathrm{C}}^{2} v=\pi Z_{\mathrm{LC}}|q Q| \frac{1-E_{\mathrm{em}} / m c^{2}}{1-E_{\mathrm{em}} / 2 m c^{2}} v=A v,
\end{aligned}
$$

where

$$
A=\pi Z_{\mathrm{LC}}|q Q| \frac{1-E_{\mathrm{em}} / m c^{2}}{1-E_{\mathrm{em}} / 2 m c^{2}}
$$

shall be called the action of the electromagnetic oscillator, $[6,11,12]$, and

$$
\begin{aligned}
Z_{\mathrm{LC}} & =\sqrt{\frac{L}{C}}=\sqrt{\frac{L^{\prime} \mathrm{d} z}{C^{\prime} \mathrm{d} z}}=\sqrt{\frac{L^{\prime}}{C^{\prime}}} \\
& =\sqrt{\frac{\mu}{\varepsilon}} \frac{\sqrt{(\ln \chi+1 / 4) \ln \left(\chi / 2+\sqrt{\chi^{2} / 4-1}\right)}}{\pi} \\
& =\sqrt{\frac{\mu}{\varepsilon}} \frac{\sigma(\chi)}{\pi}
\end{aligned}
$$

is the characteristic impedance of Lecher's line, [5], while

$$
\sigma(\chi)=\sqrt{(\ln \chi+1 / 4) \ln \left(\chi / 2+\sqrt{\chi^{2} / 4-1}\right)}
$$

shall be called the structural coefficient of Lecher's line [4].

The solution $E_{\mathrm{em}}$ of Equation (6) now reads:

$$
E_{\mathrm{em}}=\pi Z_{\mathrm{LC}}|q Q| v+m c^{2}-\sqrt{\left(\pi Z_{\mathrm{LC}}|q Q| v\right)^{2}+\left(m c^{2}\right)^{2}} \text {. }
$$

\section{Structural Constant and Action Constant}

Now, with regard to Equation (6), $E_{\mathrm{em}}=A v$, the action of electromagnetic oscillator can be written as:

$$
A=\pi Z_{\mathrm{LC}}|q Q|+\frac{m c^{2}}{v}-\sqrt{\left(\pi Z_{\mathrm{LC}}|q Q|\right)^{2}+\left(\frac{m c^{2}}{v}\right)^{2}} .
$$

Constant part of this solution, which does not depend on natural frequency $v$, we denote as action constant $A_{0}=\pi Z_{\mathrm{LC}}|q Q|$. Each oscillator has its own action constant. Equation (11) is then:

$$
A=A_{0}+m c^{2} / v-\sqrt{A_{0}^{2}+\left(m c^{2} / v\right)^{2}}
$$

and also we can now write Equations (6) and (10) as (see Planck-Einstein equation):

$$
\begin{aligned}
E_{\mathrm{em}} & =A_{0} v\left(1-E_{\mathrm{em}} / m c^{2}\right) /\left(1-E_{\mathrm{em}} / 2 m c^{2}\right), \\
E_{\mathrm{em}} & =A_{0} v+m c^{2}-\sqrt{\left(A_{0} v\right)^{2}+\left(m c^{2}\right)^{2}} \mid A_{0} v / m c^{2} \rightarrow 0 \\
& \approx A_{0} v,
\end{aligned}
$$

and with $E_{\mathrm{em}}=e V$ the first part of the Equation (13) gives (see the test of Duane-Hunt's law [4]):

$$
v=\left.\frac{e V}{A_{0}} \frac{1-e V / 2 m c^{2}}{1-e V / m c^{2}}\right|_{e V / m c^{2} \rightarrow 0} \approx \frac{e V}{A_{0}} .
$$

Also, $A_{0}$ can now be written using Equation (8):

$$
A_{0}=\pi Z_{\mathrm{LC}}|q Q|=\pi \sqrt{\frac{\mu}{\varepsilon}} \frac{\sigma(\chi)}{\pi}|e Z e|=\sqrt{\frac{\mu}{\varepsilon}} \sigma(\chi) Z e^{2} .
$$

One specific Lecher's line is dedicated to each atomic element. We can choose Lecher's line, which represents an atom of atomic number $Z$, arbitrarily. If we choose it so that the product of structural coefficient $\sigma(\chi)$ and atomic number $Z$, i.e. $\sigma(\chi) Z$ in Equation (15), is constant, so called structural constant, $s_{0}=\sqrt{\sigma(\chi) Z}$, then, providing $\varepsilon_{\mathrm{r}}=\mu_{\mathrm{r}}$, action constant $A_{0}$ will be the same for all atoms, i.e. it will be a universal constant for all atomic oscillators: $A_{0}=\sqrt{\mu / \varepsilon} s_{0}^{2} e^{2}=\sqrt{\mu_{0} / \varepsilon_{0}} s_{0}^{2} e^{2}$. The only unknown quantity in this expression is $s_{0}$. Let's see how we shall determine it.

Namely, below $\delta=2 \rho,(\chi=2)$, Lecher's line does not exist, because in that case two conductors become just one guide. Therefore, in the region below $\chi \leq 2$, Lecher's line cannot represent a single atom. However, the limit $\chi=2$ can be used to determine the structural constant $s_{0}$. For example, I estimate, [4], that only ten percent increase of $\chi$, $($ i.e. $\chi=1.1 \times 2=2.2)$, is not big enough to include all of about forty of unstable elements in this region. An increase of twenty percent of $\chi$, $(\chi=1.2 \times 2=2.4)$, however, should then include almost 40 elements, exactly from ${ }_{118}^{294}$ Uuo, $Z=118$, to the first always-stable atom of lead, ${ }_{82}^{207} \mathrm{~Pb}, Z=82$. Therefore, $s_{0}=\sqrt{\sigma(\chi) Z}=\sqrt{\sigma(2.4) 82}=\sqrt{0.837 \times 82} \approx 8.28$. Now, one can calculate $A_{0}=\sqrt{\mu_{0} / \varepsilon_{0}} s_{0}^{2} e^{2} \approx 6.63 \times 10^{-34} \mathrm{~J} \cdot \mathrm{s}$ (it is like Planck's $h$ ), and $2 s_{0}^{2} \approx 137.11$ (like fine-structure constant $1 / \alpha)$. The best agreement with the finestructure constant gives: $s_{0}=8.27756$. Then the action constant of the atomic oscillator is always the same and it 
is equal to Planck's constant $h$. This is a realistic and proven solution.

\section{The Wavelength and Momentum of the Electromagnetic Wave in an Atom}

The momentum of a limited plane electromagnetic wave, like the momentum of a photon, is related to its phase velocity by $p_{\mathrm{em}}=E_{\mathrm{em}} / u_{\mathrm{em}}=E_{\mathrm{em}} /(\lambda v)$, where $\lambda$ is the wavelength of the electromagnetic wave, and on the other hand, in accordance with the law of conservation of momentum, the linear momentum of the electron, [10], is equal to the momentum of the electromagnetic wave, (see Compton effect [9]),

$$
\frac{E_{\mathrm{em}}}{\lambda v}=\frac{m}{\sqrt{1-\beta^{2}}} v=\frac{m c \beta}{\sqrt{1-\beta^{2}}} .
$$

From Equation (16), by using Equations (4), (14) and $E_{\text {em }}=e V$, we obtain (see de Broglie wavelength; in the case of low-energy is $\mathrm{eV} / m \mathrm{c}^{2} \rightarrow 0, \mathrm{eV} \approx K \approx m v^{2} / 2$ ):

$$
\lambda=\left.\frac{A_{0}}{\sqrt{2 m e V}} \frac{\left(1-e V / m c^{2}\right)^{2}}{\sqrt{\left(1-e V / 2 m c^{2}\right)^{3}}}\right|_{e V / m c^{2} \rightarrow 0} \approx \frac{A_{0}}{m v} .
$$

Phase velocity, $\lambda v$, of electromagnetic wave from Equations (14) and (17) is

$$
\begin{aligned}
u_{\mathrm{em}} & =\lambda v=\left.\sqrt{\frac{e V}{2 m}} \frac{1-e V / m c^{2}}{\sqrt{1-e V / 2 m c^{2}}}\right|_{e V / m c^{2} \rightarrow 0} \\
& \approx \frac{v}{2} .
\end{aligned}
$$

The momentum $p_{\mathrm{em}}$ we obtain from Equations (13), (17) and (18), with $E_{\mathrm{em}}=e V$ :

$$
\begin{aligned}
p_{\mathrm{em}} & =\frac{E_{\mathrm{em}}}{u_{\mathrm{em}}}=\frac{A_{0} v}{\sqrt{\frac{e V}{2 m} \sqrt{1-e V / 2 m c^{2}}}} \mid e V / m c^{2} \rightarrow 0 \\
& \approx \frac{A_{0}}{\lambda}
\end{aligned}
$$

\section{Properties of Space in the Model of Atom}

As I have already stated at the beginning, that phase velocity is also $u_{\mathrm{em}}=1 / \sqrt{\mu \varepsilon}$, while it is now clear that the wave impedance $\sqrt{\mu / \varepsilon}$, [which is an integral part of the characteristic impedance of Lecher's line in Equation (8), and hence integral part of the action constant $A_{0}$ in Equation (15)], should therefore remain unchanged, i.e., we get a system of two equations:

$$
\begin{aligned}
& \sqrt{\mu / \varepsilon}=\sqrt{\mu_{0} / \varepsilon_{0}}, \\
& 1 / \sqrt{\mu \varepsilon}=\sqrt{e V}\left(1-e V / m c^{2}\right) / \sqrt{2 m\left(1-e V / 2 m c^{2}\right)},
\end{aligned}
$$

with two unknowns, $\mu$ and $\varepsilon$. The solutions of these equations are [6]:

$$
\begin{aligned}
& \mu=\sqrt{\frac{\mu_{0}}{\varepsilon_{0}}} \sqrt{\frac{2 m}{e V}} \frac{\sqrt{1-e V / 2 m c^{2}}}{1-e V / m c^{2}}=\mu_{\mathrm{r}} \mu_{0}, \\
& \varepsilon=\sqrt{\frac{\varepsilon_{0}}{\mu_{0}}} \sqrt{\frac{2 m}{e V}} \frac{\sqrt{1-e V / 2 m c^{2}}}{1-e V / m c^{2}}=\varepsilon_{\mathrm{r}} \varepsilon_{0}, \\
& \mu_{\mathrm{r}}=\varepsilon_{\mathrm{r}}=\frac{1}{\sqrt{\mu_{0} \varepsilon_{0}}} \sqrt{\frac{2 m}{e V}} \frac{\sqrt{1-e V / 2 m c^{2}}}{1-e V / m c^{2}} .
\end{aligned}
$$

\section{Equation Like Schrödinger's Equation}

A current value of linearly polarized standing wave reads $[3,4]$ :

$$
\begin{aligned}
& E_{\mathrm{x}}(z, t)=E_{0} \sin \left(\frac{2 \pi z}{\lambda}\right) \cos (\omega t), \\
& H_{\mathrm{y}}(z, t)=-\frac{E_{0}}{\sqrt{\mu / \varepsilon}} \cos \left(\frac{2 \pi z}{\lambda}\right) \sin (\omega t),
\end{aligned}
$$

where $E_{0}$ is the maximum value, i.e., the amplitude of electric field strength $E, E_{\mathrm{x}}(z, t)$ is the $x$-component of the electric field strength dependent on the $z$-axis and the time $t$, and $H_{\mathrm{y}}(z, t)$ is the $y$-component of the magnetic field strength $H$ dependent on the $z$-axis and the time $t$. All that we shall continue to write for the $y$-component of the magnetic field $H_{\mathrm{y}}(z, t)$ shall be applied in the exact same way to the $x$-component of the electric field $E_{\mathrm{x}}(z, t)$. If we use the second derivative of the previous equation, $H_{\mathrm{y}}(z, t)$, with respect to $z$, we get:

$\partial^{2} H_{\mathrm{y}}(z, t) / \partial z^{2}+(2 \pi / \lambda)^{2} H_{\mathrm{y}}(z, t)=0$. After the inclusion of wavelength $\lambda$, from Equation (17), we obtain:

$$
\frac{\partial^{2} H_{\mathrm{y}}(z, t)}{\partial z^{2}}+\frac{8 \pi^{2} m e V\left(1-e V / 2 m c^{2}\right)^{3}}{A_{0}^{2}\left(1-e V / m c^{2}\right)^{4}} H_{\mathrm{y}}(z, t)=0 .
$$

In the case of low-energy $\left(e V / m c^{2} \rightarrow 0, e V \approx K=E_{\mathrm{T}}-U\right)$, we have:

$$
\frac{\partial^{2} H_{\mathrm{y}}(z, t)}{\partial z^{2}}+\frac{8 \pi^{2} m}{A_{0}^{2}}\left(E_{\mathrm{T}}-U\right) H_{\mathrm{y}}(z, t)=0,
$$

which in this form resembles the non-relativistic Schrödinger's equation for a single particle moving in an electric field, [1,2]:

$$
\nabla^{2} \psi(x, y, z, t)+\frac{8 \pi^{2} m}{h^{2}}\left(E_{\mathrm{T}}-U\right) \psi(x, y, z, t)=0,
$$

where $\psi(x, y, z, t)$ is the state function, and $h$ is Planck constant, $h=A_{0}$.

This similarity of Equations (24) and (25) is not surprising, because Schrödinger's equation is based on the 
following assumptions [1]:

- For a micro-object there exists a state function, whose complex amplitude satisfies the same equation as the complex amplitude of the electromagnetic wave.

- Between the energy of the object $E_{\mathrm{T}}$ and the frequency of the wave $v$ exists relation $E_{\mathrm{T}}=h v$.

- The phase velocity of the wave corresponds to the Equation (19).

At low energies, all these assumptions are met for the electron and for the fields $\boldsymbol{E}$ and $\boldsymbol{H}$. Therefore Equation (24) actually represents Schrödinger's equation.

In the same way, using the second derivative with respect to time $t$, with $\omega=2 \pi v$, we obtain the following from the current values $H_{\mathrm{y}}(z, t)$ of linearly polarized standing wave: $\partial^{2} H_{\mathrm{y}}(z, t) / \partial t^{2}+\omega^{2} H_{\mathrm{y}}(z, t)=0$; or, using Equation (14) (in addition to $e V=-E_{\mathrm{T}}$ ),

$$
\frac{\partial^{2} H_{\mathrm{y}}(z, t)}{\partial t^{2}}+\left(\frac{2 \pi}{A_{0}} E_{\mathrm{T}} \frac{1+E_{\mathrm{T}} / 2 m c^{2}}{1+E_{\mathrm{T}} / m c^{2}}\right)^{2} H_{\mathrm{y}}(z, t)=0 .
$$

Then in the case of low-energy:

$$
\frac{\partial^{2} H_{\mathrm{y}}(z, t)}{\partial t^{2}}+\left[\frac{2 \pi}{A_{0}}\left(E_{\mathrm{T}}-U\right)\right]^{2} H_{\mathrm{y}}(z, t)=0 .
$$

\section{Conclusion}

Using Maxwell's equations we determined that the voltage and current on the transmission line as well as an electromagnetic wave in the atom are described using the same differential equations. Electromagnetic energy in the transmission line is linearly proportional to the natural frequency of oscillation of the $L C$ circuit that belongs to this line. By analogy, the same is true for the electromagnetic energy of the atom. Factor of proportionality, which we call the action of electromagnetic oscillator, however, is not constant. Because of Lorentz' theory of electrons this factor depends on the energy, or frequency, of electromagnetic wave. The part of the factor of proportionality, that is not dependent on the frequency, which we denoted as action constant, completely coincides with Planck's constant. For low-energy the wave- length of the wave in the atom corresponds to de Broglie's equation, and the frequency corresponds to DuaneHunt's law. We found that the electromagnetic wave in the atom is described by Schrödinger's equation. All of this indicates that atoms can be well described only with the help of Maxwell's equations and Lorentz' theory of electrons. All this provides a deeper entry into the matter and reveals a different view of the atom.

\section{Acknowledgements}

The Wolfram Research, Inc. Mathematica software is used by courtesy of Systemcom Ltd., Zagreb, Croatia.

\section{REFERENCES}

[1] H. Hänsel and W. Neumann, "Physik," Spektrum Akademischer Verlag, Heidelberg, 1995.

[2] H. Czichos and Association Hütte, "Die Grundlagen der Ingenieurwissenschaften," Springer-Verlag, Berlin, 1989.

[3] G. Bellotti, Advances in Natural Science, Vol. 5, 2012, pp. 7-11.

[4] M. Perkovac, "Statistical Test of Duane-Hunt's Law and Its Comparison with an Alternative Law," 2010. http://arxiv.org/abs/1010.6083

[5] J. D. Jackson, "Classical Electrodynamics," John Wiley \& Sons, Inc., New York, 1998.

[6] M. Perkovac, "Maxwell Equations for Nanotechnology," Proceedings of the 35th International Convention of the IEEE MIPRO, Opatija, 21-25 May 2012, pp. 429-436.

[7] R. H. Good, "Classical Electromagnetism," Saunders College Publishing, Fort Worth, 1999.

[8] R. Rüdenberg, "Elektrische Schaltvorgänge," Julius Springer, Berlin, 1923.

[9] D. C. Giancolli, "Physics for Scientists and Engineers," Prentice Hall, Englewood Cliffs, 1988.

[10] L. Page and N. I. Adams, "Electrodynamics," D. Van Nostrand Company, Inc., New York, 1940.

[11] M. Perkovac, Physics Essays, Vol. 15, 2002, pp. 41-60. doi:10.4006/1.3025509

[12] M. Perkovac, Physics Essays, Vol. 16, 2003, pp. 162-173. doi:10.4006/1.3025572 